\title{
Quantum Singularity of Levi-Civita Spacetimes
}

\author{
D A Konkowski†, T M Helliwell \\ $\dagger$ Department of Mathematics, U.S. Naval Academy, Annapolis, Maryland, 21402 \\ U.S.A. \\ $\ddagger$ Department of Physics, Harvey Mudd College, Claremont, California 91711 USA \\ E-mail: dak@usna.edu, T_Helliwell@HMC.edu
}

\begin{abstract}
Quantum singularities in general relativistic spacetimes are determined by the behavior of quantum test particles. A static spacetime is quantum mechanically singular if the spatial portion of the wave operator is not essentially self-adjoint. Here Weyl's limit point-limit circle criterion is used to determine whether a wave operator is essentially self-adjoint. This test is then applied to scalar wave packets in Levi-Civita spacetimes to help elucidate the physical properties of the spacetimes in terms of their metric parameters.
\end{abstract}

PACS numbers: 04.20 Dw, O4.62.+v, 03.65 Db 


\section{Introduction}

In classical general relativity, singularities are not part of the spacetime; they are boundary points indicated by incomplete geodesics or incomplete curves of bounded acceleration in a maximal spacetime (see, e.g. [1, 2]). So at least for timelike and null geodesics, this incompleteness can be considered as the abrupt ending of classical particle paths.

What happens if, instead of classical particles, one uses quantum mechanical particles to identify singularities? This is the question G. Horowitz and D. Marolf [3] set out to answer following earlier work by R. Wald [4]. They call a spacetime quantum mechanically nonsingular if the evolution of a test scalar wave packet in the spacetime, representing a quantum mechanical particle, is uniquely determined by the initial wave packet, without having to place arbitrary boundary conditions at the classical singularity. Their construction is restricted to static spacetimes.

Mathematically, this evolution is related to properties of a quantum mechanical operator. A static spacetime is quantum mechanically singular [3] if the spatial portion of the Klein-Gordon wave operator is not essentially self-adjoint [5] 6]. If this operator is not essentially self-adjoint then the evolution of a test scalar wave packet is not determined uniquely by the initial wave packet; boundary conditions at the classical singularity are needed to "pick out" the correct wave function and thus one needs to add information that is not already present in the wave operator, spacetime metric, and manifold. This will be described further in the paragraphs below.

Horowitz and Marolf restrict the wave operator to the Klein-Gordon operator, but it has been shown [7] that Maxwell and Dirac operators may also be used with equivalent generic results. Throughout this paper we will restrict the analysis to Klein-Gordon operators.

A relativistic quantum particle with mass $M$ can be described by a positive frequency solution to the Klein-Gordon equation

$$
\frac{\partial^{2} \Psi}{\partial t^{2}}=-A \Psi
$$

in a static spacetime 3]. The spatial Klein-Gordon operator $A$ is

$$
A \equiv-V D^{i}\left(V D_{i}\right)+V^{2} M^{2}
$$

where $V^{2}=-\xi_{\nu} \xi^{\nu}$ (here $\xi^{0}$ is the timelike Killing field) and $D_{i}$ is the spatial covariant derivative on the static slice $\Sigma$. The appropriate Hilbert space $H$ is $\mathcal{L}^{2}(\Sigma)$, the space of square integrable functions on $\Sigma$. This choice agrees with that of Horowitz and Marolf [3]. The volume element used to define $H$ is $V^{-1}$ times the natural volume element on $\Sigma$. We do not choose the first Sobolev norm $H^{1}$ proposed by Ishibashi and Hosoya 8]; that choice is related to Dirichlet boundary conditions at the singularity [4]. If we initially define the domain of $A$ to be $C_{0}^{\infty}, A$ is a real positive symmetric operator and self-adjoint extensions always exist [5]. If there is one unique self-adjoint extension $A_{E}$, 
then $A$ is essentially self-adjoint [5]. In this case the Klein-Gordon equation for a free relativistic particle takes the form 3 ]

$$
i \frac{\partial \Psi}{\partial t}=\left(A_{E}\right)^{1 / 2} \Psi
$$

with

$$
\Psi(t)=\exp \left(-i t\left(A_{E}^{1 / 2}\right) \Psi(0)\right.
$$

Equations (3) and (4) are ambiguous if $A$ is not essentially self-adjoint. This fact led Horowitz and Marolf [3] to define quantum-mechanically singular spacetimes as those in which $A$ is not essentially self-adjoint.

In their paper Horowitz and Marolf test several classically singular spacetimes to determine whether they are quantum mechanically singular as well. They find that Reissner-Nordström, negative mass Schwarzschild, and the $2 D$ cone remain singular when probed by quantum scalar test particles; however, certain orbifolds, extreme Kaluza-Klein black holes, the $D=5$ fundamental string, and a few other examples are nonsingular. Helliwell and Konkowski [9] and Helliwell, Konkowski and Arndt [7] show that a broad class of quasiregular spacetimes (spacetimes with quasiregular singularities) are quantum mechanically singular. This work was an extension of earlier work by Kay and Studer [10] which showed that the $2 D$ cone and a $4 D$ idealized cosmic string are not essentially self-adjoint.

The work of Horowitz and Marolf [3] and subsequent work by Helliwell, Konkowski and Arndt [7] test for essential self-adjointness of the spatial operator $A$ by using a von Neumann criterion [11, 12]. This method involves studying solutions to

$$
A \Psi= \pm i \Psi
$$

and finding the number of solutions that belong to $\mathcal{L}^{2}(\Sigma)$ for each sign of $i$. This determines the von Neumann deficiency indices which indicate whether the operator is essentially self-adjoint or whether it has self-adjoint extensions, and how many selfadjoint extensions it has $\ddagger$. If the deficiency indices are $(0,0)$, so that no solutions are square integrable, then the operator is essentially self-adjoint and so has a unique selfadjoint extension. The wave behavior described by the operator is uniquely determined for all time by the operator, the spacetime metric, and the manifold. No additional information in the form of boundary conditions need be added.

In this paper we use an alternative test for quantum singularity based upon the limit point-limit circle criterion for essential self-adjointness of operators. That is, we use a theorem of Weyl's [5] to relate the essential self-adjointness of the operator via the von Neumann deficiency indices to the "potential" which determines the behavior of the scalar wave packet. The effect is determined by the limit point-limit circle criterion which we will discuss in Section 3. After developing this technique in the context of $\ddagger$ For an introduction to the mathematics of self-adjoint operators and von Neumann deficiency indices, see [7] which is based on [5, 8, 13. 
determining the quantum singularity nature of a general spacetime, we study the socalled Levi-Civita spacetimes in particular.

Levi-Civita spacetimes are static, primarily cylindrically symmetric, spacetimes that are classically singular at " $r=0$ " unless the metric is Minkowski or Minkowski in accelerated coordinates. They are used to model infinite line masses and idealized cosmic strings. There has been some controversy in the literature about the physical relevance of certain parameters; this will be reviewed in Section 2. The quantum singularity or nonsingularity of $r=0$ will be studied in Section 4 (following the mathematical background in Section 3). Finally in Section 5 we use the quantum singularity results for certain parameter values to glean what information we can about the physical interpretation of the corresponding spacetimes.

\section{Levi-Civita Spacetimes}

The metric in Levi-Civita spacetimes [14] has the form

$$
d s^{2}=r^{4 \sigma} d t^{2}-r^{8 \sigma^{2}-4 \sigma}\left(d r^{2}+d z^{2}\right)-\frac{r^{2-4 \sigma}}{C^{2}} d \theta^{2}
$$

where $\sigma$ and $C$ are real numbers $(C>0)$. It is straightforward to show that if $\sigma$ is replaced by $1 / 4 \sigma$ in the metric, the spacetime is unchanged, although a coordinate transformation is required to make this obvious. Therefore we need only study the range $-\frac{1}{2} \leq \sigma \leq \frac{1}{2}$. Neither of the parameters $\sigma$ and $C$ can be removed by a coordinate transformation [15, 17, 16]. Computation of the Kretschmann scalar

$$
R_{\mu \nu \sigma \tau} R^{\mu \nu \sigma \tau}=\frac{64 \sigma^{2}(2 \sigma-1)^{2}}{\left(4 \sigma^{2}-2 \sigma+1\right)^{3} r^{4}}
$$

shows that $r=0$ is a scalar curvature singularity for all $\sigma$ except $\sigma=0,1 / 2$.

If $\sigma=0$, the metric is

$$
d s^{2}=-d t^{2}+d r^{2}+d z^{2}+\frac{r^{2}}{C^{2}} d \theta^{2} .
$$

If $C=1$, this is simply Minkowski spacetime in cylindrical coordinates. It thus seems reasonable to choose the coordinate ranges

$$
-\infty<t<\infty,-\infty<z<\infty, 0<r<\infty, 0 \leq \theta \leq 2 \pi
$$

with 0 and $2 \pi$ identified. If $C \neq 1$, equation 8 is the metric for an idealized cosmic string. There is a quasiregular ("disclination") singularity at $r=0$ (see, e.g., 7] for a discussion). This is a topological singularity, not a curvature singularity. It is thus reasonable to identify the parameter $C$ in the general metric, equation (6), with a topological property of the spacetime, that is, the deficit angle.

The sigma parameter is less easily interpreted [15, 17, 16]. For certain values one can interpret these spacetimes as those of infinite line masses. This interpretation is clearest for $|\sigma| \ll 1$. The most physically realistic range is $0<\sigma<1 / 4$, where $\sigma$ represents 
the mass per unit length and timelike circular orbits exist. As $\sigma$ increases from 1/4 to $1 / 2$ the interpretation is more difficult [15, 17, 16. In this range, the Kretschmann scalar decreases with increasing $\sigma$, which seems to suggest that the gravitational field is becoming weaker. But as several authors comment [15, 17, 16, the Kretschmann scalar may not be a good indicator of gravitational strength; the acceleration of test particles, increasing with increasing $\sigma$ in the interval $(1 / 4,1 / 2)$, may be a better indicator. Interior solutions matching to exterior Levi-Civita spacetimes exist for the entire range $0<\sigma<1 / 2$. Bonnor [15] proposes to interpret Levi-Civita as the spacetime generated by a cylinder whose radius increases with increasing $\sigma$ and tends to infinity as $\sigma$ tends to $1 / 2$. Thus the entire range $0<\sigma<1 / 2$ can be taken to represent an "infinite line mass".

The $\sigma=1 / 2$ case is more problematic. Bonnor's analysis suggests (but does not prove) that when $\sigma=1 / 2$ the cylinder becomes a plane, an interpretation first put forward by Gautreau and Hoffmann [18] based on different considerations. In fact, the $\sigma=1 / 2$ metric

$$
d s^{2}=-r^{2} d t^{2}+d r^{2}+d z^{2}+\frac{1}{C^{2}} d \theta^{2}
$$

is flat. We can transform to Minkowski coordinates

$$
d s^{2}=-d \bar{t}^{2}+d \bar{x}^{2}+d \bar{y}^{2}+d \bar{z}^{2}
$$

if we let $\bar{t}=r \sinh t, \bar{x}=r \cosh t, \bar{y}=\theta / C$, and $\bar{z}=z$. Here we can obviously let the $\bar{y}$ coordinate range from $-\infty$ to $\infty$. This is flat spacetime described from the view of an accelerating frame of reference. This seems to support the interpretation of the $\sigma=1 / 2$ case as a planar source producing flat spacetime described by a uniformly accelerating observer [15]. In other words, one can interpret it as the spacetime of a gravitational field produced by an infinite planar sheet of positive mass density.

On the other hand, one can interpret the metric with $\sigma=-1 / 2$ as the gravitational field produced by an infinite sheet of negative mass density (an idea also first proposed by Gautreau and Hoffmann [18]). However, this case is also problematic [15, 17, 16]. The metric

$$
d s^{2}=-\frac{1}{r^{2}} d t^{2}+r^{2}\left(d r^{2}+d z^{2}+\frac{1}{C^{2}} d \theta^{2}\right)
$$

is not flat and it has a curvature singularity at $r=0$. It has planar symmetry with one additional extra Killing vector beyond those in the general Levi-Civita spacetimes. The interpretation of this spacetime as one caused by an infinite plane of negative mass density comes from the fact that test particles are repelled from the $r=0$ plane and the fact that "the Gaussian curvature of spacelike 'eigensurfaces' is zero" [18]. This is a reasonable interpretation; the "problem" comes from interpreting both $\sigma=1 / 2$ and $\sigma=-1 / 2$ as planes of infinite (positive/negative) mass density as one is singularity-free and the other has a curvature singularity [17, 16].

We will now study the quantum singularity properties of these spacetimes for various parameter values in the following sections. We begin by introducing 
the mathematics necessary to discuss essentially self-adjoint operators and quantum singularities.

\section{Weyl's Limit Point - Limit Circle Criterion}

A particularly convenient way to establish essential self-adjointness in the spatial operator of the Klein-Gordon equation is to use the concepts of limit circle and limit point behavior. $\S$ The approach is as follows. The Klein-Gordon equation for Levi-Civita spacetimes can be separated in the coordinates $t, r, \theta, z$. Only the radial equation is nontrivial. With changes in both dependent and independent variables, the radial equation can be written as a one-dimensional Schrödinger equation

$$
H \Psi(x)=E \Psi(x)
$$

where $x \in(0, \infty)$ and the operator $H=-d^{2} / d x^{2}+V(x)$.

Definition 1 The potential $V(x)$ is in the limit circle case at $x=0$ if for some, and therefore for all E, all solutions of equation (13) are square integrable at zero. If $V(x)$ is not in the limit circle case, it is in the limit point case.

A similar definition pertains to $x=\infty$. The potential $V(x)$ is in the limit circle case at $x=\infty$ if all solutions of equation (13) are square integrable at infinity; otherwise, $V(x)$ is in the limit point case at infinity.

There are of course two linearly independent solutions of the Schrödinger equation for given $E$. If $V(x)$ is in the limit circle case at zero, both solutions are $\mathcal{L}^{2}$ at zero, so all linear combinations are $\mathcal{L}^{2}$ as well. We would therefore need a boundary condition at $x=0$ to establish a unique solution. If $V(x)$ is in the limit point case, the $\mathcal{L}^{2}$ requirement eliminates one of the solutions, leaving a unique solution without the need of establishing a boundary condition at $x=0$. This is the whole idea of testing for quantum singularities; there is no singularity if the solution is unique, as it is in the limit point case. The critical theorem is due to Weyl [5].

Theorem 1 (The Weyl limit point-limit circle criterion.) If $V(x)$ is a continuous real-valued function on $(0, \infty)$, then $H=-d^{2} / d x^{2}+V(x)$ is essentially self-adjoint on $C_{0}^{\infty}(0, \infty)$ if and only if $V(x)$ is in the limit point case at both zero and infinity.

The following theorem can be used to establish the limit circle-limit point behavior at infinity [5].

Theorem 2 (Theorem X.8 of Reed and Simon [5].) If $V(x)$ is continuous and real-valued on $(0, \infty)$, then $V(x)$ is in the limit point case at infinity if there exists a positive differentiable function $M(x)$ so that

(i) $V(x) \geq-M(x)$

$\S$ This section is based on Appendix to X.1 in Reed and Simon [5] 
(ii) $\int_{1}^{\infty}[M(x)]^{-1 / 2} d x=\infty$

(iii) $M^{\prime}(x) / M^{3 / 2}(x)$ is bounded near $\infty$.

Then $V(x)$ is in the limit point case (complete) at $\infty$.

A sufficient choice of the $M(x)$ function for our purposes is the power law function $M(x)=c x^{2}$ where $c>0$. Then (ii) and (iii) are satisfied, so if $V(x) \geq-c x^{2}, V(x)$ is in the limit point case at infinity.

A theorem useful near zero is the following.

Theorem 3 (Theorem X.10 of Reed and Simon [5].) Let $V(x)$ be continuous and positive near zero. If $V(x) \geq \frac{3}{4} x^{-2}$ near zero then $V(x)$ is in the limit point case. If for some $\epsilon>0, V(x) \leq\left(\frac{3}{4}-\epsilon\right) x^{-2}$ near zero, then $V(x)$ is in the limit circle case.

These results can now be used to help test for quantum singularities in the Levi-Civita spacetimes.

\section{Limit Point - Limit Circle properties of Levi-Civita Spacetimes}

The Klein-Gordon equation for a scalar particle of mass $M$ is

$$
\square \Phi \equiv g^{\mu \nu} \Phi_{\mu \nu}+\frac{1}{\sqrt{g}}\left(\sqrt{g} g^{\mu \nu}\right)_{, \nu} \Phi_{\mu}=M^{2} \Phi ;
$$

for the Levi-Civita metrics it is

$$
-r^{-4 \sigma} \Phi_{, t}+r^{8 \sigma^{2}+4 \sigma}\left(\Phi_{, r r}+\Phi_{, z z}\right)+C^{2} r^{4 \sigma-2} \Phi_{, \theta \theta}+r^{-8 \sigma^{2}+4 \sigma-1} \Phi_{, r}=M^{2} \Phi .
$$

The equation separates using $\Phi=e^{-i \omega t} e^{i k z} e^{i m \theta} R(r)$, where $\omega, k$ are continuous and $m=0, \pm 1, \pm 2, \cdots$. The resulting radial equation is

$$
\frac{d^{2} R}{d r^{2}}+\frac{1}{r} \frac{d R}{d r}+\left[\omega^{2} r^{8 \sigma^{2}-8 \sigma}-k^{2}-M^{2} r^{8 \sigma^{2}-4 \sigma}-m^{2} C^{2} r^{8 \sigma^{2}-2}\right] R=0 .
$$

As described in Section 1, square integrability is judged by evaluating the integral

$$
I=\int d r \sqrt{g_{3} / g_{00}} R^{*} R=(1 / C) \int d r r^{8 \sigma^{2}-8 \sigma+1} R^{*} R
$$

where $g_{3}$ is the negative determinant of the 3-space portion of the metric tensor [4, 3]. The resulting integral is invariant under coordinate transformations.

To test for limit point-limit circle behavior one must rewrite the radial equation in the one-dimensional Schrödinger-equation form

$$
\frac{d^{2} \Psi(x)}{d x^{2}}+[E-V(x)] \Psi=0
$$

for which square integrability is judged by the integral $I=\int d x \Psi^{*} \Psi$. Except for the special case $\sigma=1 / 2$, which we return to later, the appropriate transformations of dependent and independent variables are $R=(1 / \sqrt{x}) \Psi$ and $r=\left(\alpha C x^{2}\right)^{1 /(2 \alpha)}$ where $\alpha \equiv(2 \sigma-1)^{2}$. 
Equation (16) transforms to equation (18) if we let $E=C \omega^{2} / \alpha$ and

$$
\begin{aligned}
V(x) & =\left(C k^{2} / \alpha\right)\left(\alpha C x^{2}\right)^{(-\alpha+1) / \alpha}+\left(C M^{2} / \alpha\right)\left(\alpha C x^{2}\right)^{2 \sigma / \alpha} \\
& +\left(m^{2} C^{3} / \alpha\right)\left(\alpha C x^{2}\right)^{(4 \sigma-1) / \alpha}-1 /\left(4 x^{2}\right) .
\end{aligned}
$$

In the limit $x \rightarrow \infty$ the final term vanishes, leaving a non-negative $V(x)$. Therefore the solutions are limit point at infinity for all $\sigma(\sigma \neq 1 / 2)$, since as $x \rightarrow \infty, V(x)>-c x^{2}$ for any positive constant $c[5,6]$.

It remains to test the solutions in the limit $x \rightarrow 0$. This we do by identifying the important terms in $V(x)$. The final term diverges as $x^{-2}$. The first term in $V(x)$ diverges less quickly for any finite $\sigma$. The second term $\sim x^{4 \sigma / \alpha}$, whose exponent has the minimum value $-1 / 2$ at $\sigma=-1 / 2$. The third term $\sim x^{2(4 \sigma-1) / \alpha}$, whose exponent has the minimum value -2 at $\sigma=0$.

Therefore unless $\sigma=0$ (or $\sigma=1 / 2$, which we treat later), the equation has the form

$$
\frac{d^{2} \Psi}{d x^{2}}+\frac{1}{4 x^{2}} \Psi=0
$$

as $x \rightarrow 0$. By Theorem 3 the solutions should exhibit limit circle behavior; in fact, one solution is $\Psi_{1}=x^{1 / 2}$ and the other is $\Psi_{2}=\Psi_{1} \int^{x} d x / \Psi_{1}^{2}=x^{1 / 2} \ln (x)$. Both are $\mathcal{L}^{2}$ near $x=0$, so are indeed limit circle.

If $\sigma=0$, the equation has the form

$$
\frac{d^{2} \Psi}{d x^{2}}-\frac{m^{2} C^{2}-1 / 4}{x^{2}} \Psi=0
$$

as $x \rightarrow 0$. One solution is $\Psi_{1}=x^{1 / 2+|m| C}$; the other is $\Psi_{2}=x^{1 / 2} \ln (x)$ (if $\left.m=0\right)$, or $\Psi_{2}=x^{1 / 2-|m| C}$ (if $m \neq 0$ ). Both solutions are limit circle if $|m| C<1$, and limit point if $|m| C \geq 1$.

In the special case $\sigma=0, C=1$, the spacetime is Minkowski with no classical singularity at $r=0$. In that case $x=r$ and $R_{1}=1, R_{2}=\ln (r)$. The solution $R_{2}=\ln (r)$ is unacceptable in this case because it diverges at $r=0$ even though $r=0$ is a regular hypersurface in the spacetime. $\|$ If $\sigma=0, C \neq 1$ the spacetime corresponds to a linear idealized cosmic string with a quasiregular singularity at $r=0$.

For the special case $\sigma=1 / 2$ the radial Klein-Gordon equation is

$$
\frac{d^{2} R}{d r^{2}}+\frac{1}{r} \frac{d R}{d r}+\left[-\left(k^{2}+M^{2}+m^{2} C^{2}\right)+\omega^{2} / r^{2}\right] R=0 .
$$

The transformation $R(r)=\Psi(x)$ and $r=\exp (C x)$ puts the equation in the form

$$
\frac{d^{2} \Psi}{d x^{2}}+[E-V(x)] \Psi=0
$$

where $E=C^{2} \omega^{2}$ and

$$
V(x)=C^{2}\left(k^{2}+M^{2}+m^{2} C^{2}\right) \exp (2 C x)
$$

$\|$ We assumed at the outset that the domain of the spatial operator consists of smooth functions with compact support away from the origin. For Minkowski space the points $r=0$ are arbitrary, so it is unphysical to permit a function that diverges at $r=0$ and nowhere else. 
with $\int d r \sqrt{g_{3} / g_{00}} R^{*} R=\int d x \Psi^{*} \Psi$.

In the transformation $r=0$ corresponds to $x=-\infty$, while $r=\infty$ corresponds to $x=\infty$. So in this case we must determine the limit point-limit circle behavior at $x= \pm \infty$. In this case $V(x)>-c x^{2}$ at both $\pm \infty$, for any positive $c$. Therefore the solutions are limit point at both ends. It is interesting that the $\sigma=1 / 2$ case is a special case which must be studied on the interval $(-\infty, \infty)$. This result seems to support Bonnor's description of the $\sigma=1 / 2$ case as a cylinder whose radius tends to $\infty$ as $\sigma$ tends to $1 / 2$.

\section{Conclusions}

In the preceding section we showed that the Klein-Gordon operator is limit point at infinity for the whole class of Levi-Civita spacetimes. If $\sigma$ is neither zero nor one-half, the Klein-Gordon operator is limit circle at $r=0$ and thus not essentially self-adjoint, so all $\sigma \neq 0, \sigma \neq 1 / 2$ Levi-Civita spacetimes are quantum mechanically singular as well as being classically singular.

If $\sigma=0$ and $C=1$, the spacetime is simply Minkowski space. One of the two solutions of the radial Klein-Gordon equation can be rejected because it diverges at a regular point $(r=0)$ of the spacetime. The operator is therefore limit point at $r=0$, and so Minkowski spacetime is quantum mechanically nonsingular (a well known fact, repeated here for completeness).

par If $\sigma=0$ and $C \neq 1$, the spacetime is the conical spacetime corresponding to an idealized cosmic string. The operator is limit circle if $|m| C<1$ and limit point if $|m| C \geq 1$. Therefore the cosmic string spacetimes are quantum mechanically singular for azimuthal quantum number $m$ such that $|m| C<1$ and nonsingular if $|m| C \geq 1$. If arbitrary values of $m$ are allowed, these spacetimes are quantum mechanically singular in agreement with earlier results [9, 7, 10]. Recall that these spacetimes are also classically singular with a quasiregular ("disclination") singularity at $r=0$.

If $\sigma=1 / 2$ the classical spacetime is flat and without a classical singularity. We have shown that this spacetime is quantum mechanically nonsingular as well for massive scalar particles obeying the Klein-Gordon equation, since the operator is limit point at both ends and thus essentially self-adjoint.

Thus for the Levi-Civita spacetimes, all that are classically singular are also quantum mechanically singular, and all that are classically nonsingular $(\sigma=0, C=1$, and $\sigma=1 / 2$ ) are also quantum mechanically nonsingular. The classically and quantummechanically nonsingular spacetimes correspond to isolated values of $\sigma$, so that (for example) even though the spacetime $\sigma=0, C=1$ is nonsingular, the spacetimes with $\sigma \rightarrow 0, C=1$ are singular. The only discrepency between classical and quantum singularities are for the $\sigma=0, C \neq 1$ modes with $|m| C \geq 1$, which are quantum mechanically nonsingular in a classically singular spacetime. The physical reason is that the wavefunction for large values of $m$ in a flat space with a quasiregular singularity at $r=0$ are unable to detect the presence of the singularity because of the repulsive 
centrifugal potential $m^{2} C^{2} / x^{2}$ in equation (21). The limit point/limit circle criterion, together with the theorems that connect it to the effective potential in a Schrodinger equation, provide physical insight into when quantum singularities are prevented from occurring by potential barriers.

\section{Acknowledgments}

We thank David Clarke, Jack Harrison and Cassidi Reese for useful conversations. One of us (DAK) was partially funded by NSF grant PHY99-88607 to the U.S. Naval Academy. She also thanks Queen Mary, University of London, where some of this work was carried out.

\section{References}

[1] Hawking S W and Ellis G F R 1973 The Large-Scale Structure of Spacetime (Cambridge: Cambridge University Press)

[2] Ellis G F R and Schmidt B G 1977 Gen. Relativ. Grav 8915

[3] Horowitz G T and Marolf D 1995 Phys. Rev. D 525670

[4] Wald R M 1980 J. Math Phys. 212802

[5] Reed M and Simon B 1972 Functional Analysis (New York: Academic Press) Reed M and Simon B 1972 Fourier Analysis and Self-Adjointness (New York: Academic Press)

[6] Richmyer R -D 1978 Principles of Advanced Mathematical Physics, Vol. I (New York: Springer)

[7] Helliwell T M, Konkowski D A and Arndt V 2003 Gen. Relativ. Grav. 3579

[8] Ishibashi A and Hosoya A 1999 Phys. Rev. D 60104028

[9] Konkowski D A and Helliwell T M 2001 Gen. Relativ. Grav. 331131

[10] Kay B S and Studer U M 1991 Commun. Math. Phys. 139103

[11] von Neumann J 1929 Math. Ann. 10249

[12] Weyl H 1910 Math. Ann. 68220

[13] Bonneau G, Faraut J and Valent G 2001 Am. J. Phys. 69322

[14] Levi-Civita T 1919 Rend. Acc. Lincei 28101

[15] Bonnor W B "The static cylinder in general relativity" in On Einstein's Path ed. A. Harvey 1999 (New York: Springer) 113

[16] Herrera L, Ruifernández J and Santos N O 2001 Gen. Relativ. Grav. 33515

[17] Herrera L, Santos N O, Teixeira A F F, and Wang A Z 2001 Class. Quantum Grav. 183847

[18] Gautreau R and Hoffmann R B 1969 Nuovo Cimento B 61411 